\title{
Facile Synthesis of Straight Polyaniline Nanostick in Hydrogel
}

Linghui Meng a, Yan Lu ${ }^{\mathrm{b}}$, Xudong Wang ${ }^{\mathrm{a}}$, Jian Zhang ${ }^{\mathrm{a}}$, Yueqin Duan ${ }^{\mathrm{b}}$, Chenxi $\mathrm{Li}^{*^{\mathrm{a}}}$

a. Key Laboratory of Functional Polymer Materials, Ministry of Education.

Institute of Polymer Chemistry, Nankai University, Tianjin, 300071, China.

b. Institute of Material Physics, Tianjin University of Technology, Tianjin 300191, China

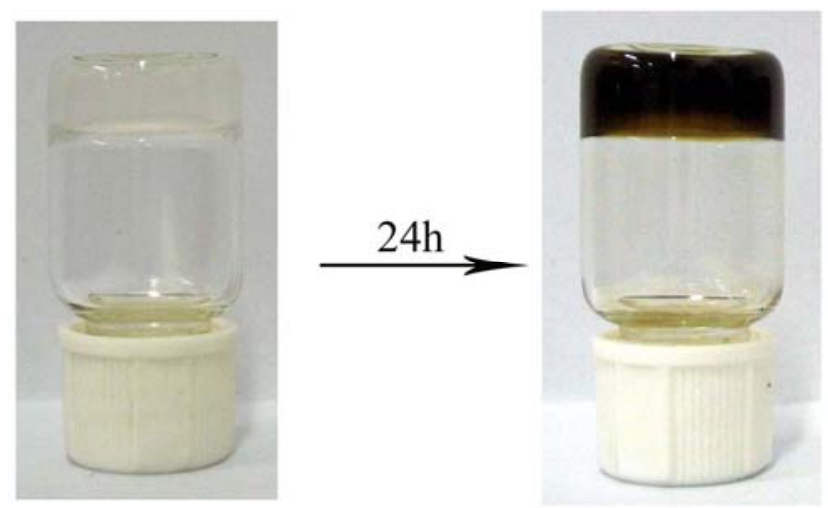

Figure S1. Snapshots showing color change of PANI synthesized in hydrogel from transparent to dark brown.

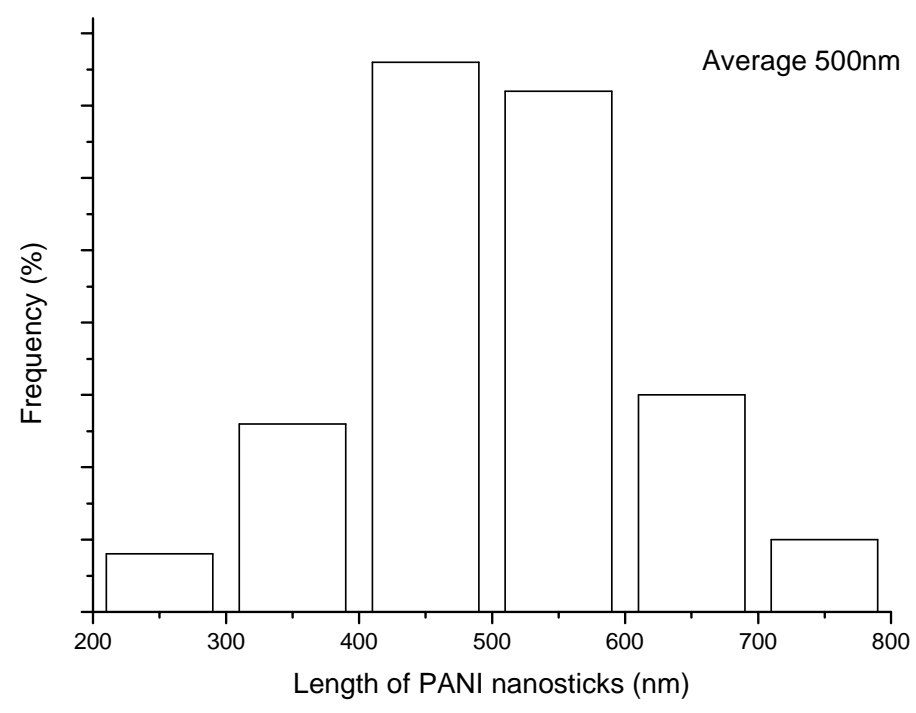

Figure S2. Length distribution analysis of PANI nanosticks. 


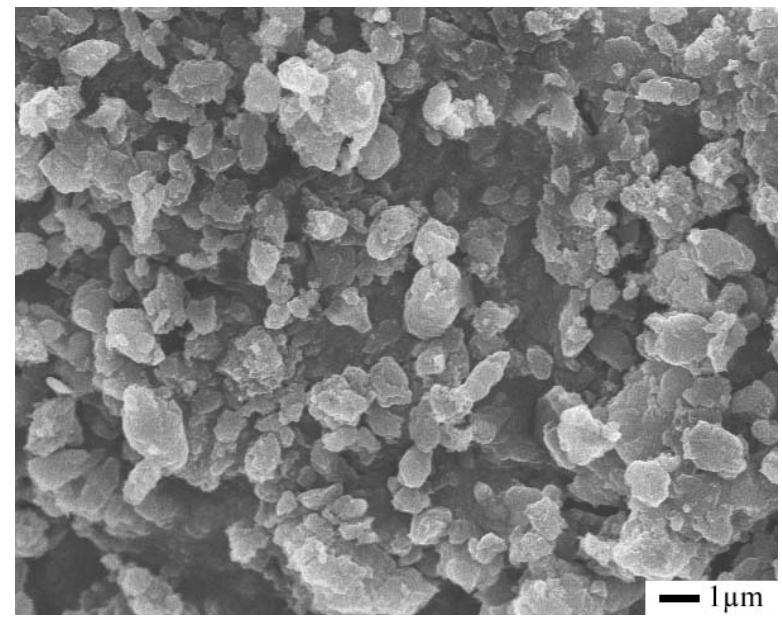

Figure S3. SEM image of PANI bulks synthesized under similar reaction conditions only without hydrogelator.
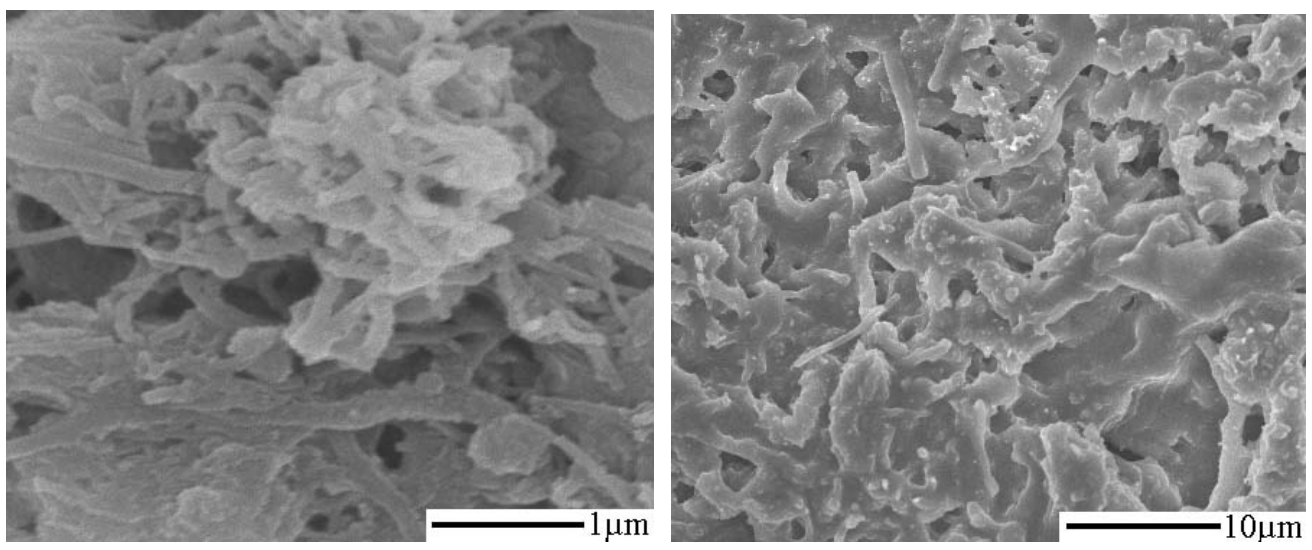

Figure S4. Effect of concentration of hydrogelator on the morphology of PANI.

SEM images of PANI synthesized using CA-PEG400: 10mg (left) and 30mg (right)
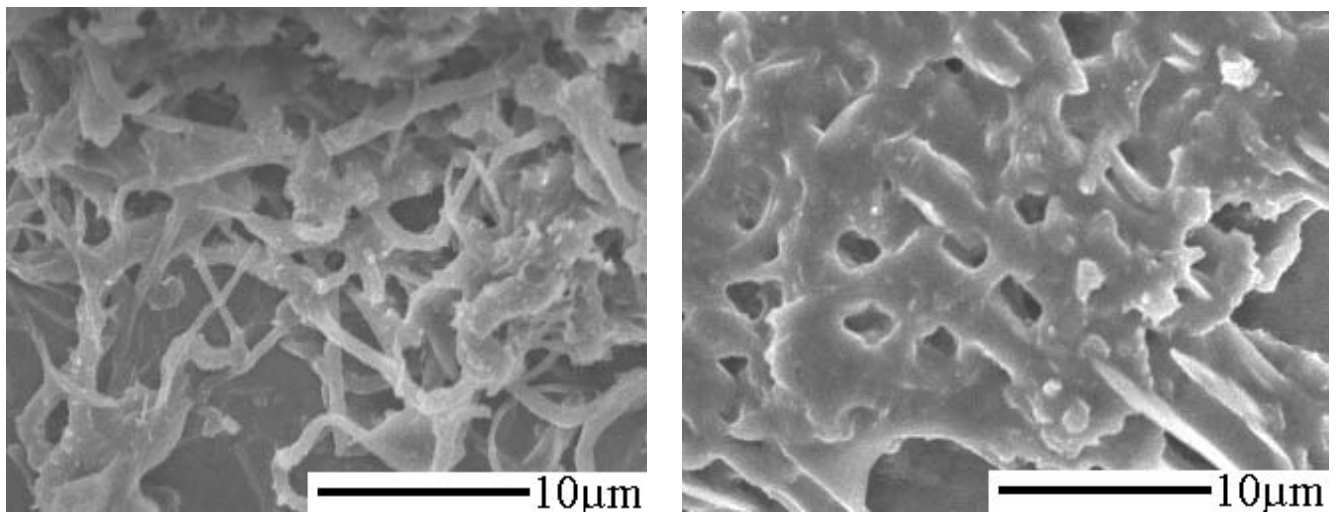

Figure S5. Effect of concentration of oxidant on the morphology of PANI.

SEM images of PANI synthesized using aqueous solution of APS: 0.05M (left) and 0.2M (right) 